\title{
Relation Between Blood Resistivity and Hematocrit in Fresh Human Fetal Blood
}

\author{
KENNETH SANDBERG, ${ }^{(13)}$ BENGT-ARNE SJÖQVIST, AND TORSTEN OLSSON \\ Department of Pediatrics I, University of Göteborg, and Research Laboratory of Medical Electronics, Chalmers \\ University of Technology, Göteborg, Sweden
}

\begin{abstract}
Summary
This study determined the relation between hematocrit and resistivity of fetal blood and compared it with values obtained in similar studies on adult blood. Both exponential and MaxwellFrick-estimated relationships were calculated and compared. The results indicate that there is no significant difference between resistivity in adult and fetal blood. The best relation between blood resistivity and fetal hematocrit is obtained by using the MaxwellFrick estimated curve calculated in the following manner:
\end{abstract}

$$
\rho=53.0(1+\xi \cdot h) /(1-h)
$$

where

$$
\begin{gathered}
\xi=1.2 \\
h=H / 100(H=\text { hematocrit }) .
\end{gathered}
$$

\section{Speculation}

Hematocrit varies over a wide range during the newborn period. Accurate knowledge of resistivity-hematocrit relationships over the entire hematocrit range is essential whenever blood resistivities are to be estimated from hematocrit values. This study reveals that a better correlation is obtained when using the Maxwell-Frick model instead of the ordinary exponential model. Thus, a tool is offered to improve the accuracy of the impedance cardiography method in newborns.

The need for noninvasive methods in measuring physiologic and pathophysiologic conditions and courses in the newborn baby is evident.

Impedance cardiography, one method to measure the cardiac stroke volume in healthy adults, was developed by Kubicek et al. (5). The ability of the method to measure pulmonary blood flow in the presence of left to right shunt in children was described by Lababidi et al. (6). The cardiac stroke volume is calculated according to the formula

$$
\mathrm{SV}=\rho \frac{\mathrm{L}^{2}}{\mathrm{z}_{0}^{2}} \mathrm{dz} / \mathrm{dt} \cdot \mathrm{T}
$$

developed by Kubicek et al. where SV = cardiac stroke volume in $\mathrm{ml} ; \rho=$ blood resistivity in $\mathrm{ohm} \mathrm{cm} ; \mathrm{L}=$ distance between the detecting inner electrodes in $\mathrm{cm} ; \mathrm{z}_{0}=$ basal transthoracic impedance in ohm; dz/dt $=$ minimum first derivative in ohm $/ \mathrm{s} ; \mathrm{T}=$ ventricular ejection time in sec. The resistivity, $\rho$, is a material constant. Considering blood as a suspension of blood cells in plasma, overall resistivity can be expressed in terms of one, resistivity and volume relation of the cells and plasma and, two, a factor dependent on the shape of the suspended particles $(2,7$, 12). Thus, there are theoretical reasons behind expressing blood resistivity as a function of hematocrit. There is a wider range of hematocrit levels in neonates than in adults. Hematocrit values exceeding $60 \%$ are not unusual in newborns (9). There is also a continuous change in hematocrit during the newborn period be- cause of the adaption to extrauterine life. It is therefore necessary to take into account the actual hematocrit of the newborn baby when trying to measure the thoracic circulatory events by means of impedance cardiography described by Kubicek et al.

Measurement of resistivity in adult blood with varying hematocrit was published by Geddes and Sadler (4) and Axenborg (1). Mohapatra et al. (8) published resistivity data from blood samples partly obtained from placenta. Their values differ from data on human adult blood resistivity. Results relating only to resistivity measurements on fresh human placental blood are not published.

\section{MATERIALS AND METHODS}

A conductivity cell filled with human fetal umbilical cord blood, never older than $24 \mathrm{hr}$, was used for the measurements. The cord blood was collected immediately after delivery. To avoid clotting, heparin $(5000 \mathrm{IU} / \mathrm{ml})$ was added to the blood samples to yield an amount of $1 \mathrm{ml} / 10 \mathrm{ml}$ blood. This amount of heparin had no significant influence on the resistivity (see below). The conductivity cell was placed in a water-bath maintained at $37^{\circ} \mathrm{C}$ and rotated to prevent the cells from settling. To make sure that the blood sample had reached a stable temperature, resistivity values were recorded only after remaining stable for at least $5 \mathrm{~min}$. The low hematocrit values were created by diluting the blood samples with plasma from adults.

The conductivity cell (Fig. 1) is the same as the one described by Axenborg (1) and essentially the same as the one described by Geddes and Da Costa (3). It consists of a syringe equipped with two-plane parallel stainless steel electrodes, one at the end of the syringe (A) and the other affixed to the barrel (B). The syringe is also equipped with two Luer-connections, which allow filling and expulsion of the electrolyte. Resistance is measured between the electrodes at two different spacings, and the resistivity is calculated by the formula

$$
\rho=\mathrm{A} \Delta \mathrm{Z} / \Delta \mathrm{L}
$$

where $\mathrm{A}$ is the cross-sectional area, $\Delta \mathrm{Z}$ the change in impedance, and $\Delta \mathrm{L}$ is the change in length. This technique eliminates the influence from electrode polarization which would otherwise give values that are too high.

The change in impedance is measured with the impedance meter described by Olsson et al. (10). This impedance meter operates at a frequency of $50 \mathrm{kHz}$ and a current of $0.2 \mathrm{~mA}$ peak to peak. At the frequency used in the impedance meter, the impedance of blood is almost completely resistive $(10,11)$.

The results are based upon 10 original blood samples, each consisting of mixed fetal blood of the same blood group collected from different placentas. These samples were diluted with plasma to obtain various hematocrit values, making up a total of 34 measuring points.

To make sure that a small amount of heparin did not affect the resistivity, we did the following investigation. Different amounts of heparin were added to a certain amount of blood. The hematocrit was kept at the same value in the different blood samples. This was done by replacing plasma with the actual amount of heparin. 


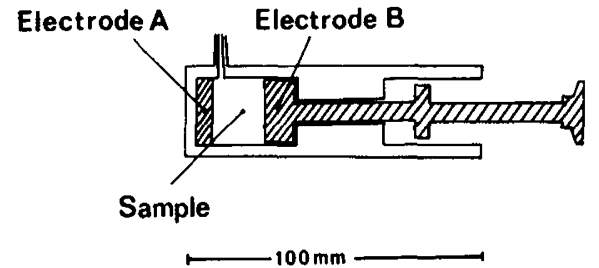

Fig. 1. The conductivity cell used in the resistivity measurements.

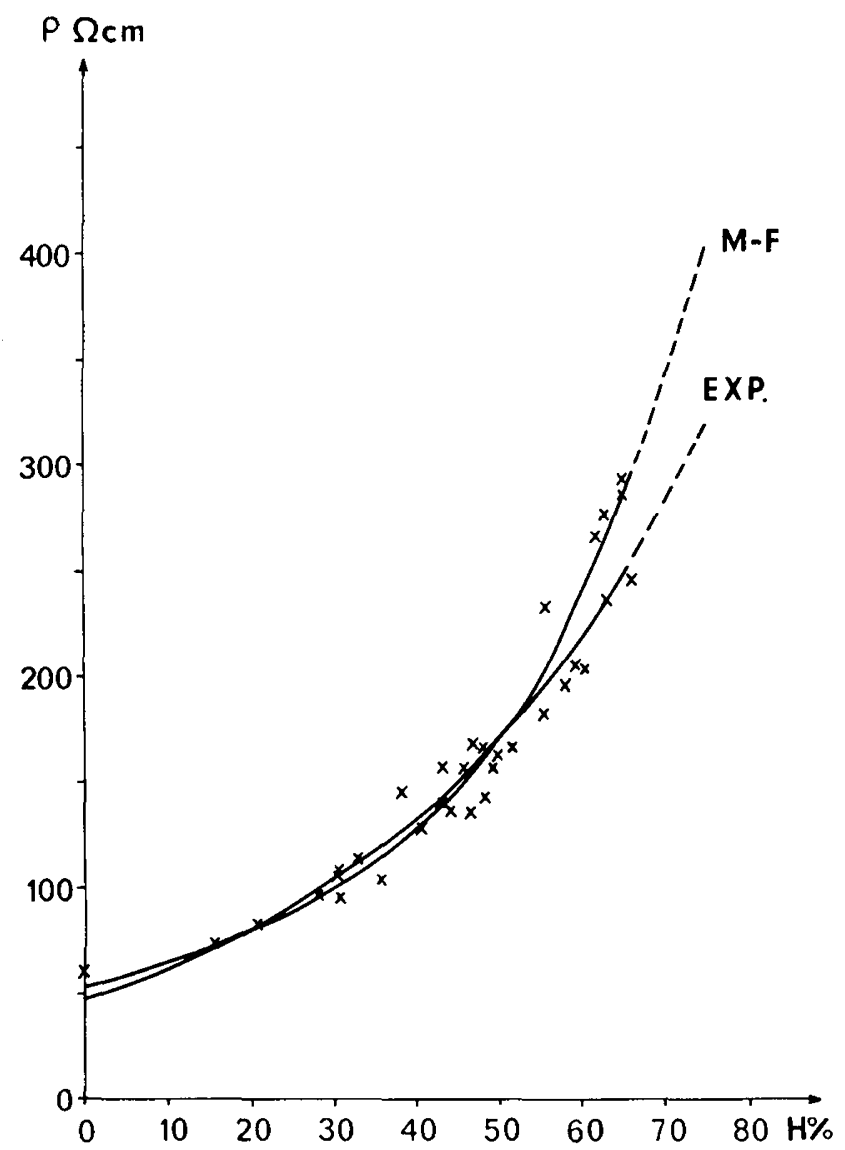

Fig. 2. Relationship between blood resistivity $(\rho)$ and hematocrit $(\mathrm{H})$ Original observations, exponential ( $E X P$ ), and Maxwell-Frick estimated $(M-F)$ curves represented.

\section{RESULTS}

The relationship between the blood resistivity $\rho$ and hematocrit $\mathrm{H}$ can be seen in Figure 2. A least-squares fit of the measured data to an exponential function is provided by the relation:

$$
\rho=49.477 \cdot \mathrm{e}^{0.0248 H}
$$

with a residual error of variation of 351.17 , corresponding to a standard error of 3.21. This relation is represented in Figure 2.

The measured data were also compared to the relationship described by Maxwell and Frick $(2,7,12)$. This expression deals with the resistivity of a medium in which particles of different resistivity are suspended. The particles are described by a form factor $(\xi)$ which varies according to their shape $(\xi=0.5$ for spheres). The expression has the form

$$
\rho=\rho_{\mathrm{p}}(1+\xi \mathrm{h}) /(1-\mathrm{h}),
$$

where

$$
\begin{aligned}
\rho & =\text { blood resistivity; } \\
\rho_{\mathrm{p}} & =\text { blood plasma resistivity; } \\
\mathrm{h} & =\mathrm{H} / 100 ; \mathrm{H}=\text { hematocrit. }
\end{aligned}
$$

A least squares fit of the measured data to the Maxwell-Frick equation is provided by the parameter choice:

$$
\rho_{p}=53.0 \Omega \mathrm{cm}, \xi=1.2,
$$

with a residual error of variation of 275.07 , corresponding to a standard error of 2.84. This relationship is also represented in Figure 2. In this figure, the original observations are marked as well.

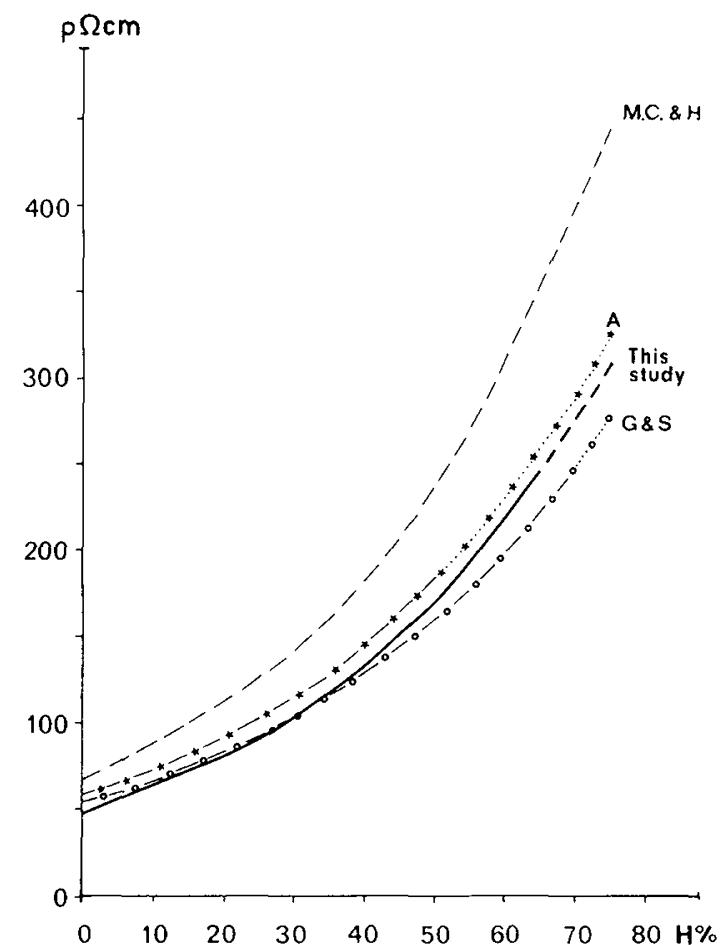

Fig. 3. Relationship between blood resistivity $(\rho)$ and hematocrit $(H)$. Exponential estimated curves by different authors: Axenborg $(A)$, Geddes and Sadler $(G \& S)$, Mohapatra et al. (M., $C \& H$.), and this study.

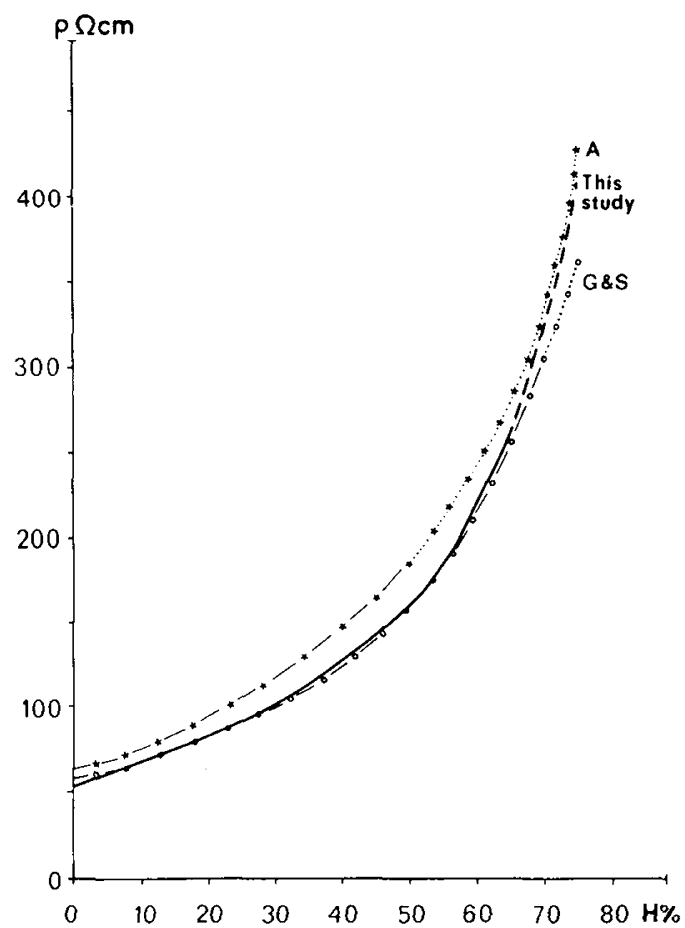

Fig. 4. Relationship between blood resistivity $(\rho)$ and hematocrit $(\mathbf{H})$. Maxwell-Frick estimated curves by different authors: Axenborg $(A)$, Geddes and Sadler $(G \& S)$, and this study. 
Table 1. Results of heparin investigation.

\begin{tabular}{c|c|c|c}
\hline $\begin{array}{c}\text { HEPARIN } \\
\text { ML. }\end{array}$ & $\begin{array}{c}\text { HEPARIN } \\
\text { VOI\% }\end{array}$ & $\begin{array}{c}H \\
\%\end{array}$ & \begin{tabular}{c}
$\rho_{C M}$ \\
\hline 0
\end{tabular} \\
\hline 1 & 0 & 35 & 94 \\
2 & 8 & 35 & 94 \\
3 & 16 & 35 & 90 \\
4 & 25 & 35 & 90 \\
5 & 33 & 35 & 87 \\
& 42 & 35 & 87
\end{tabular}

The results were compared with those obtained by other investigators. Both exponential and Maxwell-Frick estimations were compared (Figs. 3 and 4).

Replacing plasma with heparin had an effect on the resistivity values of the same order as the standard error of the fitted curve only when the heparin level was higher than the actual level in the measurement series. Hence, no correction for heparin was done (Table 1).

\section{DISCUSSION}

Impedance cardiography in the newborn period is a simple and attractive noninvasive method. It measures circulatory events in the thorax of the baby. The clinical value of the method in the neonatal period is still to be evaluated. To obtain reliable values of stroke volume according to Kubicek, it is necessary to have relevant values of the blood resistivity. This is especially important for measurements on newborn babies because of the wide range of hematocrit values. Previously published results on blood resistivity measurements are based upon few, if any, blood samples with hematocrit more than $50 \%$.

Although the resistivity is dependent on the temperature (1), the syringe was kept in a water-bath of temperature $37^{\circ} \mathrm{C}$, and measurement was made when the resistivity had maintained a stable value for $5 \mathrm{~min}$. In our investigations, we measured resistivity at a frequency of $50 \mathrm{kHz}$. Earlier investigations were carried out at other frequencies, but it has been described by several investigators $(10,11)$ that frequencies between 1 and $800 \mathrm{kHz}$ make no significant difference on the resistivity.

Our values do not differ significantly from adult blood samples values published in previous reports $(1,3)$. However, these earlier results were based mainly on measurements of hematocrit below $50 \%$ which is normal for adult blood. Our results are based on measurements over a greater range and are therefore more reliable in the upper range. The measurements were also carried out using just fetal blood.

The wide range of measured resistivity values at high hematocrit levels is most likely the net effect of several different factors. Due to the exponential shape of the resistivity-hematocrit relation, errors in the hematocrit level measurements have a much larger impact at higher levels. An uneven distribution of the blood cells in the sample will, again due to the exponential shape, have a greater influence on the measurement as the hematocrit levels increase. At very high levels, it is more difficult to keep an even distribution and prevent the cells from settling.

There is one report about blood resistivity measurements made on fetal blood. This is published by Mohapatra et al. (8). These results also include measurements of adult blood samples. Their data differ significantly from our results and all other published values of blood resistivity. This can only be explained by the use of different techniques in measuring the blood resistivity.

This study shows that the best correlation between hematocrit and blood resistivity is obtained by using the Maxwell-Frick estimated curve. Therefore, it is recommended that this curve be used whenever blood resistivities are to be estimated from hematocrit values.

\section{REFERENCES AND NOTES}

1. Axenborg, J.: An electric impedance method for measurement of aortic crosssectional area. Technical Report 5:79, Res. Lab. Med. Electr. (Chalmers University of Technology, Göteborg, Sweden, 1979).

2. Frick, H.: A mathematical treatment of the electric conductivity and capacity of disperse systems. I. The electric conductivity of a suspension of homogeneous spheroides. Physiol. Rev., 24: 575 (1924).

3. Gedes, L. A., and Da Costa, C. P.: The specific resistance of canine blood at body temperature. IEEE Trans. Biomed. Eng. (Berl.), BME-20: 51 (1973).

4. Geddes, L. A., and Sadler, C.: The specific resistance of blood at body temperature. Med. Biol. Eng., 11: 336 (1973).

5. Kubicek, W. G. et al:: Development and evaluation of an impedance cardiac output system. Aerosp. Med., 37: 1208 (1966).

6. Lababidi, Z. et al.: Evaluation of impedance cardiac output in children. Pediatrics, 47: 870 (1971).

7. Maxwell, J. C.: A Treatise on Electricity and Magnetism. Vol. 1, Ed. 3, pp. 310314 (Dover Publications, New York, 1891).

8. Mohapatra, S. N., Costeloe, K., and Hill, D. W.: Blood resistivity and its implications for the calculation of cardiac output by thoracic electrical impedance technique. Intensive Care Med., 3: 63 (1977)

9. Oh, W., and Lind, J.: Venous and capillary haematocrit in newborn infants and placental transfusion. Acta Paediatr. Scand., 55: 38 (1966)

10. Olsson, T., Daily, W., and Victorin, I.: Transthoracic impedance. Theoretical considerations and technical approach. Acta Paediatr. Scand. Suppl., 207: 15 (1970).

11. Schwan, H. P., and Kay, C. F.: Capacitive properties of body tissues. Circ. Res., 5: 439 (1957).

12. Velick, S., and Gorin, M.: The electrical conductance of suspensions of ellipsoides and its relation to the study of erythrocytes. J. Gen. Physiol., 23: 753 (1940).

13. Requests for reprints should be addressed to: K. Sandberg, Department of Pediatrics, Östra sjukhuset, S-416 85 Göteborg, Sweden.

14. This research was supported by the Swedish Medical Research Council, Project B80-19X-05703-01.

15. Received for publication June 24, 1980.

16. Accepted for publication October $23,1980$. 